\title{
THE EFFECT OF A REFRACTORY PERIOD ON THE POWER SPECTRUM OF NEURONAL DISCHARGE*
}

\author{
JOEL FRANKLIN ${ }^{\dagger}$ AND WYETH BAIR ${ }^{\ddagger}$
}

\begin{abstract}
The interspike intervals in steady-state neuron firing are assumed to be independently and identically distributed random variables. In the simplest model discussed, each interval is assumed to be the sum of a random neuron refractory period and a statistically independent interval due to a stationary external process, whose statistics are assumed known. The power spectral density (hence the autocorrelation) of the composite neuron-firing renewal process is derived from the known spectrum of the external process and from the unknown spectrum of the neuron-refraction process. The results are applied to spike trains recorded in a previous study [2] of single neurons in the visual cortex of an awake monkey. Two models are demonstrated that may produce peaks in the power spectrum near $40 \mathrm{~Hz}$.
\end{abstract}

Key words. neuronal spike trains, refractory period, autocorrelation, power spectrum, $40 \mathrm{~Hz}$ oscillation

AMS subject classifications. 92B20, 60G10, 92B05

1. Introduction. There has been recent interest in the use of the Fourier power spectrum for analyzing temporal structure in trains of action potentials recorded from neurons [5], [1]. This is largely due to the reports of stimulus-induced oscillation near $40 \mathrm{~Hz}$ in neural activity recorded in the visual cortex of the anesthetized cat [4], [6], [7], [5] and the awake monkey [9].

Bair et al. [1], using data recorded in an earlier study by Britten et al. [2], compute power spectra of single neuron spike trains. Using the power spectra, they find that most of the spike trains are well modeled by a Poisson shot-noise process modulated by a refractory period where the shots are either $\delta$-functions or boxcar functions, representing individual action potentials or bursts (temporal clusters) of action potentials, respectively. In both cases, the refractory period is implemented in the model using a Gaussian depression in the renewal density function (see [11]) for the shot-noise process, and the results of Champeney [3] are used to compute the resulting power spectrum.

In the present work, instead of relying on the renewal density to model the refractory period, we begin with a function that is explicitly designed to model the absolute and relative refractory period of the neuron. This density function describes the neuron's stochastic dead time following an action potential and is the first of two contributions to the period between successive action potentials. The remaining contribution depends on the underlying process describing the input to the cell. We allow this input process to be any stationary renewal process, not just the Poisson process used by Bair et al. [1]. Using the refraction function and the probability density function for the intervals of the underlying process, we can derive the power spectrum.

* Received by the editors November 18, 1993; accepted for publication (in revised form) May 14, 1994.

$\dagger$ Applied Mathematics Department, California Institute of Technology, Pasadena, California 91125.

$\ddagger$ Computation and Neural Systems Program, California Institute of Technology, Pasadena, California 91125 (wyeth@klab.caltech.edu). The reseach of this author was supported by the L.A. Hanson Foundation through Prof. Christof Koch and by a grant from the Office of Naval Research to Christof Koch. 
The results allow quantitative comparisons of neuronal spike trains to a variety of stochastic models. In particular, they enable us to determine how much of the regularity in successive interspike intervals in a spike train can be explained by a renewal process with a refractory period or to what extent it is necessary to assume some underlying oscillatory mechanism to explain regularity. In addition, because our model distinguishes between the contribution to the interspike interval from refraction and the contribution from input to the neuron, we believe that it provides a framework for separately characterizing a neuron's refractory period and providing a stochastic description of its inputs.

We apply the mathematical results to neuronal spike trains recorded in a previous study [2] to demonstrate four points. 1. A fixed stochastic description of a refractory period is adequate to describe the output of some neurons even when the input stimulation and the output firing rate vary dramatically. 2. The assumption of a renewal process for the generation of interspike intervals is consistent with data. 3. Spike patterns such as bursts are accounted for by the theory and result in predictable peaks in the power spectra of spike trains that are not due to regular timing. 4. If spike rate (or burst rate) becomes fast relative to the refractory period duration, peaks may develop in the power spectrum near the inverse of the peak in the interval density, and these peaks are the result of regular timing.

Many others have studied the effect of refraction on neuronal spike train statistics. Teich, Matin, and Cantor [14] consider the model of a Poisson process modified by a variable dead time which is very similar to that used by Bair et al. [1] and which is encompassed in the theory developed here. Teich and Diament [13] modeled the relative refractory period using a gradual recovery function. Related mathematical models concerning the power spectra of impulse processes appear in Lukes [10] and Heiden [8].

2. Mathematical model. We will apply the following model in $\S 3$ and will derive the mathematical assertions in $\S 4$.

We will analyze the steady-state firing of a single neuron. We shall suppose that the output potential is a renewal process

$$
x(t)=\sum_{k=-\infty}^{+\infty} h\left(t-t_{k}\right)
$$

where the $t_{k}$ are the successive firing instants and where $h\left(t-t_{k}\right)$ is the output of the single firing at the instant $t_{k}$. The function $h(t)$ is given. The ensemble $\left\{t_{k}\right\}$ is random. The successive positive lags $\tau_{k}=t_{k}-t_{k-1}$ are assumed to be statistically independent with the same p.d.f. (probability-density function) $f(\tau)$ for $\tau>0$. Although the successive lags $\tau_{k}$ are random, their p.d.f. $f(\tau)$ is assumed to be known.

We suppose that $f(\tau)$ is continuous for $\tau \geq 0$ except for isolated simple jump discontinuities, and we will assume that $f(\tau)$ has finite moments

$$
\int_{0}^{\infty} \tau^{k} f(\tau) d t
$$

for $k=0,1,2$, and preferably also for $k=3$ and 4 . We assume that the wave form $h(t)$ has a finite Fourier transform

$$
H(\omega)=\int_{-\infty}^{\infty} h(t) e^{-i \omega t} d t \quad(-\infty<\omega<\infty)
$$


We do allow $h(t)$ to be the Dirac $\delta$-function. We expect, but do not require, that $h(t) \equiv 0$ for $t<0$.

To study neuron refraction, we shall hypothesize a simple model for the successive lags $\tau_{k}=t_{k}-t_{k-1}$. We shall suppose that each lag $\tau_{k}$ is the sum of two parts

$$
\tau_{k}=\tau_{k_{1}}+\tau_{k_{2}}
$$

where $\tau_{k_{1}}$ arises from refraction, and where $\tau_{k_{2}}$ arises from all other causes, for example, from an assumed random input potential.

We shall suppose that the parts $\tau_{k_{1}}$ and $\tau_{k_{2}}$ are statistically independent, with probability densities $f_{1}(\tau)$ and $f_{2}(\tau)$ that are independent of $k$. Thus, the sum $\tau$ has the p.d.f.

$$
f(\tau)=f_{1}(\tau) * f_{2}(\tau)=\int_{0}^{\tau} f_{1}(\tau-\lambda) f_{2}(\lambda) d \lambda .
$$

The ensemble of random variables $\left\{\tau_{k_{j}}\right\}$ is supposed to be statistically independent for $-\infty<k<\infty$ and $j=1,2$.

We assume that the random variables $\tau_{k_{1}}$ and $\tau_{k_{2}}$ have finite means $\mu_{1}, \mu_{2}$ and finite variances $\sigma_{1}^{2}$ and $\sigma_{2}^{2}$. Then the total lags $\tau_{k}=\tau_{k_{1}}+\tau_{k_{2}}$ have the common mean $\mu=\mu_{1}+\mu_{2}$ and the common variance $\sigma^{2}=\sigma_{1}^{2}+\sigma_{2}^{2}$.

We will show how the mean $\mu$ and the variance $\sigma^{2}$ appear in the power spectral density of the steady-state neuron output potential $x(t)$.

The power spectral density $S_{x}(\omega)$ is defined as the Fourier transform of the autocorrelation $R_{x}(\tau)$ :

$$
S_{x}(\omega)=\int_{-\infty}^{\infty} R_{x}(\tau) e^{-i \omega \tau} d \tau
$$

where the autocorrelation $R_{x}(\tau)$ is defined as the expected value

$$
R_{x}(\tau)=\mathrm{E}[x(t) x(t-\tau)] \quad(-\infty<\tau<\infty)
$$

The autocorrelation is independent of $t$, by the assumption that the ensemble of firing instants $\left\{t_{k}\right\}$ is a stationary random process.

We will prove that $x(t)$ has the power spectral density

$$
S_{x}(\omega)=|H(\omega)|^{2} \nu\left\{2 \pi \nu \delta(\omega)+1+2 \operatorname{Re} \frac{\hat{f}(i \omega)}{1-\hat{f}(i \omega)}\right\}
$$

where $\nu$ is the average firing frequency $\nu=\mu^{-1}$, and where $\hat{f}(s)$ is the Laplace transform of the lag p.d.f:

$$
\hat{f}(s)=\int_{0}^{\infty} f(\tau) e^{-s \tau} d \tau \quad(\operatorname{Re} s \geq 0)
$$

We shall derive the limit

$$
\lim _{\omega \rightarrow 0} 2 \operatorname{Re} \frac{\hat{f}(i \omega)}{1-\hat{f}(i \omega)}=\nu^{2} \sigma^{2}-1
$$

and we will use this value at $\omega=0$ in equation (8) for $S_{x}(\omega)$. Then we may write

$$
S_{x}(\omega)=|H(\omega)|^{2} \nu\left\{2 \pi \nu \delta(\omega)+\nu^{2} \sigma^{2}+o(1)\right\} \text { as } \omega \rightarrow 0 .
$$


Here $o(1)$ is the generic symbol for some function of $\omega$ that tends to zero as $\omega \rightarrow 0$; if the lags $\tau$ have finite fourth moment $\mathrm{E}\left(\tau^{4}\right)$, we can even show that

$$
S_{x}(\omega)=|H(\omega)|^{2} \nu\left\{2 \pi \nu \delta(\omega)+\nu^{2} \sigma^{2}+O\left(\omega^{2}\right)\right\} \text { as } \omega \rightarrow 0 .
$$

In any case, the lag mean and variance, $\mu=\nu^{-1}$ and $\sigma^{2}$, appear in the power spectral density $S_{x}(\omega)$ at small frequencies, $\omega$. For the contribution of refraction and other, independent causes, we now recall the formulas

$$
\mu=\mu_{1}+\mu_{2}, \quad \sigma^{2}=\sigma_{1}^{2}+\sigma_{2}^{2},
$$

where the refraction lag $\tau_{k_{1}}$ has mean and variance $\mu_{1}$ and $\sigma_{1}^{2}$.

Example 1. To see how these results might be used, suppose that the random refraction lags $\tau_{k_{1}}$ have a gamma distribution with the common p.d.f.

$$
f_{1}(\tau)=\frac{b^{a}}{\Gamma(a)} \tau^{a-1} e^{-b \tau} \quad(\tau \geq 0)
$$

where $a$ and $b$ are unknown positive constants. Then

$$
\int_{0}^{\infty} \tau^{k} f_{1}(\tau) d \tau=a(a+1) \cdots(a+k-1) b^{-k} \quad(k=0,1,2, \ldots) .
$$

Then the first and second moments are $\mu_{1}=a b^{-1}$ and $a(a+1) b^{-2}$. Since the second moment must equal the variance plus the square of the mean, we find

$$
\mu_{1}=a b^{-1} \text { and } \sigma_{1}^{2}=a b^{-2}
$$

for the common mean and variance of the refraction lags $\tau_{j_{1}}(-\infty<j<\infty)$.

For instance, if $\mu_{1}$ is fixed and if $\sigma_{1} \rightarrow+0$, then $f_{1}(\tau)$ becomes just $\delta\left(\tau-\mu_{1}\right)$.

Suppose the total lag $\tau_{j}$ is the sum of the refraction lag $\tau_{j_{1}}$ and the independent source lag $\tau_{j_{2}}$, where the source lag has the Poisson p.d.f.

$$
f_{2}(\tau)=\nu_{2} e^{-\nu_{2} \tau} \quad(\tau \geq 0)
$$

Then the source lags $\tau_{j_{2}}$ have the common mean and variance

$$
\mu_{2}=\nu_{2}^{-1} \text { and } \sigma_{2}^{2}=\nu_{2}^{-2} \text {. }
$$

Therefore, the total lags $\tau_{j}$ have the common mean and variance

$$
\mu=a b^{-1}+\nu_{2}^{-1} \text { and } \sigma^{2}=a b^{-2}+\nu_{2}^{-2} .
$$

The p.d.f. $f(\tau)$ is the convolution $f_{1}(\tau) * f_{2}(\tau)$. The Laplace transform $\hat{f}(s)$ is the product

$$
\hat{f}(s)=\hat{f}_{1}(s) \hat{f}_{2}(s)=\left(\frac{b}{b+s}\right)^{a} \cdot \frac{\nu_{2}}{\nu_{2}+s} \quad(\operatorname{Re} s \geq 0) .
$$

To compute the power spectral density of the neuron output potential $x(t)$, we must use the expression

$$
G(\omega) \equiv 2 \operatorname{Re} \frac{\hat{f}(i \omega)}{1-\hat{f}(i \omega)} \quad(-\infty<\omega<\infty)
$$


where $G(0)$ is defined as the limit as $\omega \rightarrow 0$, which we will derive in Lemma 4.1:

$$
\begin{gathered}
G(0)=\nu^{2} \sigma^{2}-1=\mu^{-2} \sigma^{2}-1, \\
G(0)=\left(\mu_{1}+\mu_{2}\right)^{-2}\left(\sigma_{1}^{2}+\sigma_{2}^{2}\right)-1 .
\end{gathered}
$$

The full power spectral density is

$$
S_{x}(\omega)=|H(\omega)|^{2} \nu[2 \pi \nu \delta(\omega)+1+G(\omega)] .
$$

For $\omega$ near zero, we recall the assertion

$$
S_{x}(\omega)=|H(\omega)|^{2} \nu\left[2 \pi \nu \delta(\omega)+\nu^{2} \sigma^{2}+0\left(\omega^{2}\right)\right] .
$$

For instance, if the neuron-firing wave form is just the Dirac $\delta$-function $h(t)=\delta(t)$, then $H(\omega) \equiv 1$, and so

$$
S_{x}(\omega)=\nu\left[2 \pi \nu \delta(\omega)+\nu^{2} \sigma^{2}+0\left(\omega^{2}\right)\right] \text { as } \omega \rightarrow 0 .
$$

From experimental data, we can find the power spectrum $S_{x}(\omega)$. If the graph of $S_{x}(\omega)$ near $\omega=0$ indicates that

$$
S_{x}(\omega)=A \delta(\omega)+C+o(1) \text { as } \omega \rightarrow 0,
$$

then we may observe the spike strength, $A$, and the constant $C$. Knowing $A$ and $C$, we may write the equations

$$
A=2 \pi \nu^{2}, \quad C=\nu^{3} \sigma^{2}
$$

or, equivalently,

$$
A=\frac{2 \pi}{\left(\mu_{1}+\mu_{2}\right)^{2}}, \quad C=\frac{\sigma_{1}^{2}+\sigma_{2}^{2}}{\left(\mu_{1}+\mu_{2}\right)^{3}}
$$

Now, if we know the mean $\mu_{2}$ and the variance $\sigma_{2}^{2}$ of the source lags, we can solve the two equations for the mean $\mu_{1}$ and the variance $\sigma_{1}^{2}$ of the refraction lags.

By the way, to obtain the lag mean and variance, $\mu$ and $\sigma^{2}$, we do not need the power spectral density. If we observe a long sequence of firing instants $t_{0}<t_{1}<t_{2}<$ $\cdots<t_{N}$, then we may approximate the mean and variance by the empirical mean and variance,

$$
\bar{\tau}=\frac{\tau_{1}+\cdots+\tau_{N}}{N}=\frac{t_{N}-t_{0}}{N}
$$

and

$$
s^{2}=\frac{1}{N-1} \sum_{j=1}^{N}\left(\tau_{j}-\bar{\tau}\right)^{2}
$$

where $\tau_{j}=t_{j}-t_{j-1}$.

More general models of neuron firing lags. We have assumed that each firing lag $\tau_{j}$ is the sum $\tau_{j_{1}}+\tau_{j_{2}}$, where $\tau_{j_{1}}$ comes from refraction and $\tau_{j_{2}}$ comes from independent sources. We have supposed that the variates $\tau_{j_{1}}, \tau_{j_{2}}(-\infty<j<\infty)$ are statistically 
independent, where each variate $\tau_{j_{1}}$ has p.d.f. $f_{1}(\tau)$ and each variate $\tau_{j_{2}}$ has p.d.f. $f_{2}(\tau)$.

More generally, each lag $\tau_{j}$ may need to be considered as the sum of $m$ parts:

$$
\tau_{j}=\tau_{j_{1}}+\cdots+\tau_{j_{m}} \quad(-\infty<j<\infty) .
$$

Since we still have a stationary renewal process, the lags $\left\{\tau_{j}\right\}$ are still independently, identically distributed (i.i.d.), with a common p.d.f. $f(\tau)(\tau \geq 0)$.

Suppose that the partial lag $\tau_{j_{r}}$ has p.d.f. $f_{r}(\tau)(r=1, \ldots, m)$. Let the partial lags have expected values and variances

$$
\mu_{r}=\mathrm{E}\left(\tau_{j_{r}}\right) \quad \text { and } \quad \sigma_{r}^{2}=\mathrm{E}\left(\tau_{j_{r}}-\mu_{r}\right)^{2} \quad(r=1, \ldots, m) .
$$

For $m=2$ we assumed that the partial lags were independent, so that the total lag $\tau_{j}$ had mean and variance

$$
\mu=\mu_{1}+\cdots+\mu_{m} \quad \text { and } \quad \sigma^{2}=\sigma_{1}^{2}+\cdots+\sigma_{m}^{2} .
$$

Of course, these formulas remain true for $m>2$ if the partial lags are independent and the Laplace transform $\hat{f}(s)$ of the p.d.f. $f(\tau)$ is just the product of the Laplace transforms $\hat{f}_{r}(s)$ :

$$
\hat{f}(s)=\hat{f}_{1}(s) \cdots \hat{f}_{m}(s) .
$$

Even if $m=2$, these formulas must be changed if the partial lags $\tau_{j_{1}}, \ldots, \tau_{j_{m}}$ are dependent. Then we have

$$
\mu=\mu_{1}+\cdots+\mu_{m} \quad \text { but } \quad \sigma^{2}=\sum_{\alpha=1}^{m} \sum_{\beta=1}^{m} \sigma_{\alpha \beta},
$$

where $\left\{\sigma_{\alpha \beta}\right\}$ are the covariances; and now the Laplace transform $\hat{f}(s)$ generally cannot be expressed as the product $\hat{f}_{1}(s) \cdots \hat{f}_{m}(s)$.

3. Application. We will now apply the results stated in the previous section to neuronal spike trains and will use the equations presented in Example 1 of the previous section as our model. This application makes four points: 1. a fixed stochastic description of a refractory period is adequate to describe the output of some neurons even when the input stimulation and the output firing rate vary dramatically; 2 . the assumption of a renewal process for the generation of interspike intervals is often valid; 3. spike patterns such as bursts are accounted for by the theory and result in predictable peaks in the power spectra of spike trains that are not due to regular timing; and 4. if spike rate (or burst rate) becomes fast relative to the refractory period duration, peaks develop in the power spectrum at near the inverse of the peak in the interval density, and these peaks are the result of regular timing.

3.1. Methods. We analyze spike trains from a previous study [2] that were recorded extracellularly from single neurons in the motion area, MT, of the visual cortex of behaving monkeys. The visual stimulus is a $2 \mathrm{sec}$ duration dynamic random dot display in which one parameter, the motion coherence $c$, is varied from trial to trial for a particular neuron. For $c=0$, dots are plotted randomly. For $c=0.5$, half of the dots carry a motion signal in the direction that best excites the neuron being recorded, and for $c=1$, all of the dots translate in the neuron's preferred direction. 
For $c<0$, the motion signal is 180 degrees opposed to the neuron's preferred direction. See Britten et al. [2] for an exact description of the stimulus.

The occurrence times of action potentials recorded during the stimulus are digitized at a $1 \mathrm{kHz}$ resolution and represented as a function of discrete time having the values 0 and 1, where 1 represents the presence of an action potential. A segment of a spike train is shown across the top of Fig. 1. In all analysis presented here, the first $336 \mathrm{msec}$ of the neuronal response during the $2 \mathrm{sec}$ stimulus are discarded to avoid processing the early transient portion of the response.

The probability density function for the time between consecutive spikes is estimated from the neuronal data by the interspike interval (ISI) histogram. ISI histograms show the percentage of all intervals at each discrete time length (integer values in $\mathrm{msec}$ ) and are plotted using bars (see Fig. 1, left side).

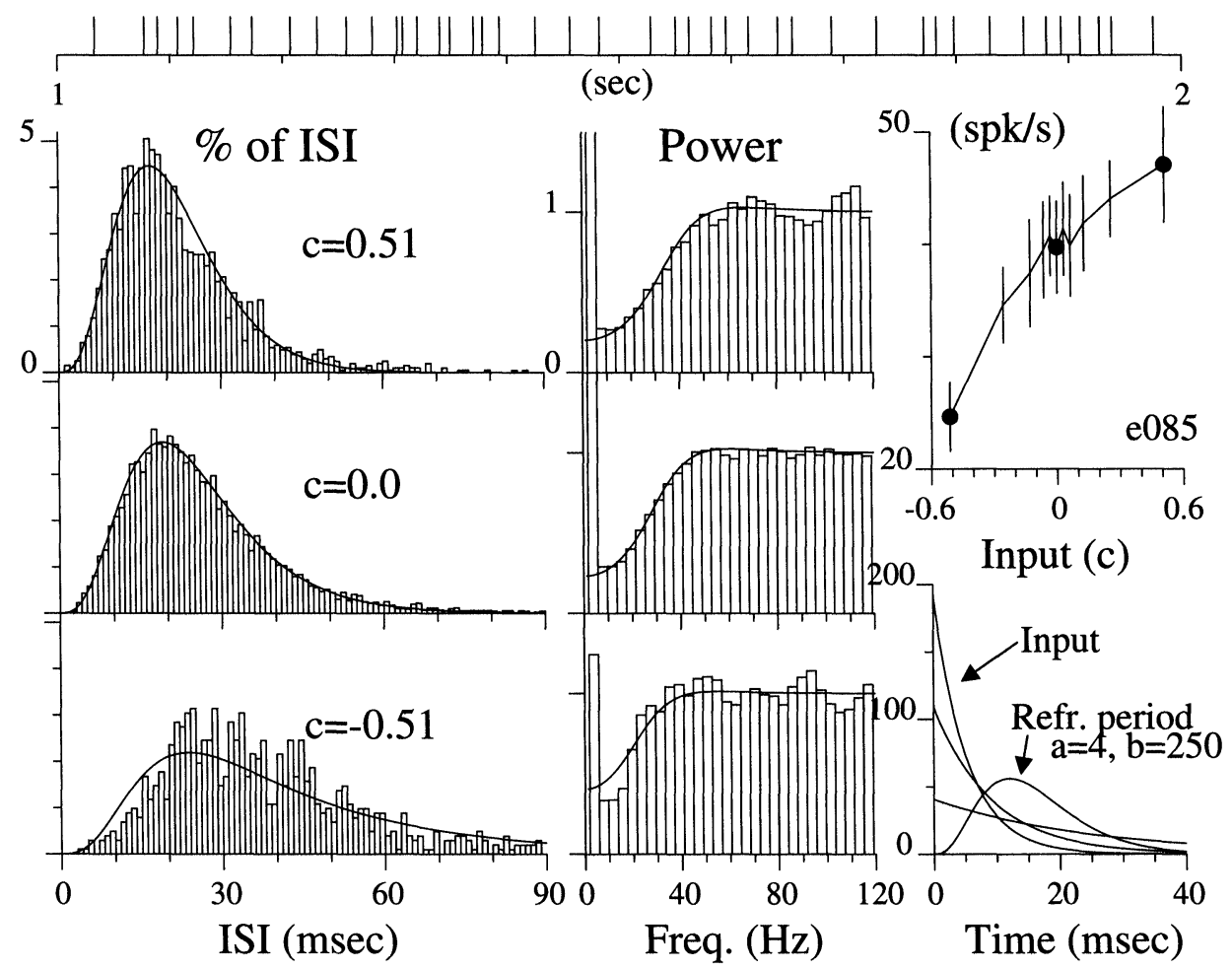

FIG. 1. Neuronal data vs. theory assuming a fixed refractory period as firing rate changes for cell e085. The top trace shows a 1 second segment of a spike train recorded at $c=0$ stimulation. The ISI histogram (left column, bars) and the neuronal power spectrum (center column, bars) were computed from $2 \mathrm{sec}$ duration trials (120 trials at $c=0 ; 30$ trials at $c= \pm 0.51)$ with the first 336 msec, containing on-transients, discarded. The upper right plot shows the output firing rate of the neuron over the range of input levels tested, and the points mark the cases that are studied here (error bars show standard deviation). Analytical curves for $f(\tau)$ (combine (5), (14), and (17)) were fitted by eye to the ISI histograms for all 11 input levels plotted in the upper right panel under the constraint that $a$ and $b$ are fixed. For a particular $a$ and $b$, the parameter $\nu_{2}$ was set for each input level so that the overall mean firing rate $\nu$ matched the values shown for the cell. The lower right plot shows the refraction density, $f_{1}(\tau)$ (see (14)), and input densities, $f_{2}(\tau)$ (see (17)), for the fit. Here $a=4.0, b=250.0$, and the values for $\nu_{2}$ may be read from the plot as the maximum values, at $\tau=0$, of the exponential densities. Analytical curves for $\tilde{S}_{x}(\omega)($ see $(100))$ based on the parameters from the ISI fits are superposed in the center column. $(h(t)$ is taken to be the $\delta$-function.) 
The Fourier power spectrum $S_{x}(\omega)$ is estimated for neuronal spike trains by computing a discrete one-sided power spectrum using a standard FFT routine and data windowing with a triangular (Parzen) window on overlapping data segments (for details, see Press et al. [12]). The neuronal power spectra are plotted with bars of width $3.9 \mathrm{~Hz}$ (resulting from $256 \mathrm{msec}$ duration data segments), the abscissa is truncated before the $500 \mathrm{~Hz}$ cutoff frequency (half of the sampling frequency) to expand the nonflat region of the spectrum, and the zero-frequency bar is always omitted. All power spectra are normalized so that the ordinate value 1.0 corresponds to the mean neuronal firing rate. This normalization is analogous to dividing (8) by $\nu$. The analytical curves superposed on neuronal power spectra in the figures are described by $\tilde{S}_{x}(\omega)$, a normalized version of $S_{x}(\omega)$, which is defined in the Appendix.

3.2. Results. We use the model presented in Example 1 of the previous section, which defines each interspike interval to be the sum of a refractory component with a gamma density and an input component with an exponential density. Given the lack of spatial and temporal correlation in the stimulus signal, it is reasonable to first model the neuron input component with the exponential density, consistent with a Poisson process.

For a given neuron, we determine the values of the refractory period parameters $a$ and $b$ which give the best fit by eye to the series of ISI histograms at all $c$ values available. Note that $a$ and $b$ do not vary as a function of the input $c$-only $\nu_{2}$ is allowed to vary, but is constrained so that

$$
\mu_{\mathrm{est}}(c)=a b^{-1}+\nu_{2}^{-1}(c),
$$

where $\mu_{\text {est }}$ is the measured mean spike rate as a function of input strength $c$ (Fig. 1, upper right) and the notation $\nu_{2}(c)$ indicates the input dependence of $\nu_{2}$. Figure 1 shows the ISI histograms and their fits $f(\tau)$ (left column) for $a=4.0, b=250.0$ (where $a / b$ is the mean ISI in seconds and $a / b^{2}$ is the variance) at the three $c$ values indicated by dots in the upper right plot. The lower right panel of Fig. 1 shows the refractory period density, $f_{1}(\tau)$, and the three input densities, $f_{2}(\tau)$, for the fit parameters. Roughly half of 58 cells examined showed fits qualitatively similar to that shown here.

The center column of Fig. 1 shows the measured power spectra of the neuronal spike trains (bars) and $\tilde{S}_{x}(\omega)$ (smooth curve) based on the parameters $a, b, \nu_{2}(c)$ (see (100) in the Appendix for an explicit formula for $\tilde{S}_{x}(\omega)$ ). The refractory period causes a dip in the power spectra at low frequencies - without the refractory period, the spectrum, that of Poisson impulses, would be flat.

Figure 2 shows the results of applying a similar analysis to a neuron that has a shorter refractory period $(a / b=4.7 \mathrm{msec}$ rather than $16 \mathrm{msec})$ and a wider range of output firing rates (upper right panel). It is striking that the left (rising) sides of the ISI histograms are well fit by the same fixed refractory period ( $a=8, b=1700$, thus $\mu_{1}=4.7 \mathrm{msec}, \sigma_{1}=1.7 \mathrm{msec}$ ) over a broad range of spike rates, particularly for $c=1.0$, where the firing rate appears, based on the upper right plot (Fig. 2), to be saturated. The analytical power spectra (smooth curves on center column plots) predict the shape change observed in the neuronal power spectra (Fig. 2, bar plots, center column) as $c$ changes. Again, the values of the theoretical inputs, $\nu_{2}$, are observed as the $y$-intercept of the decaying exponentials in the lower right plot. Some small systematic errors appear in the fits, in particular, the right sides of the ISI histograms tend to have faster than exponential fall off. 


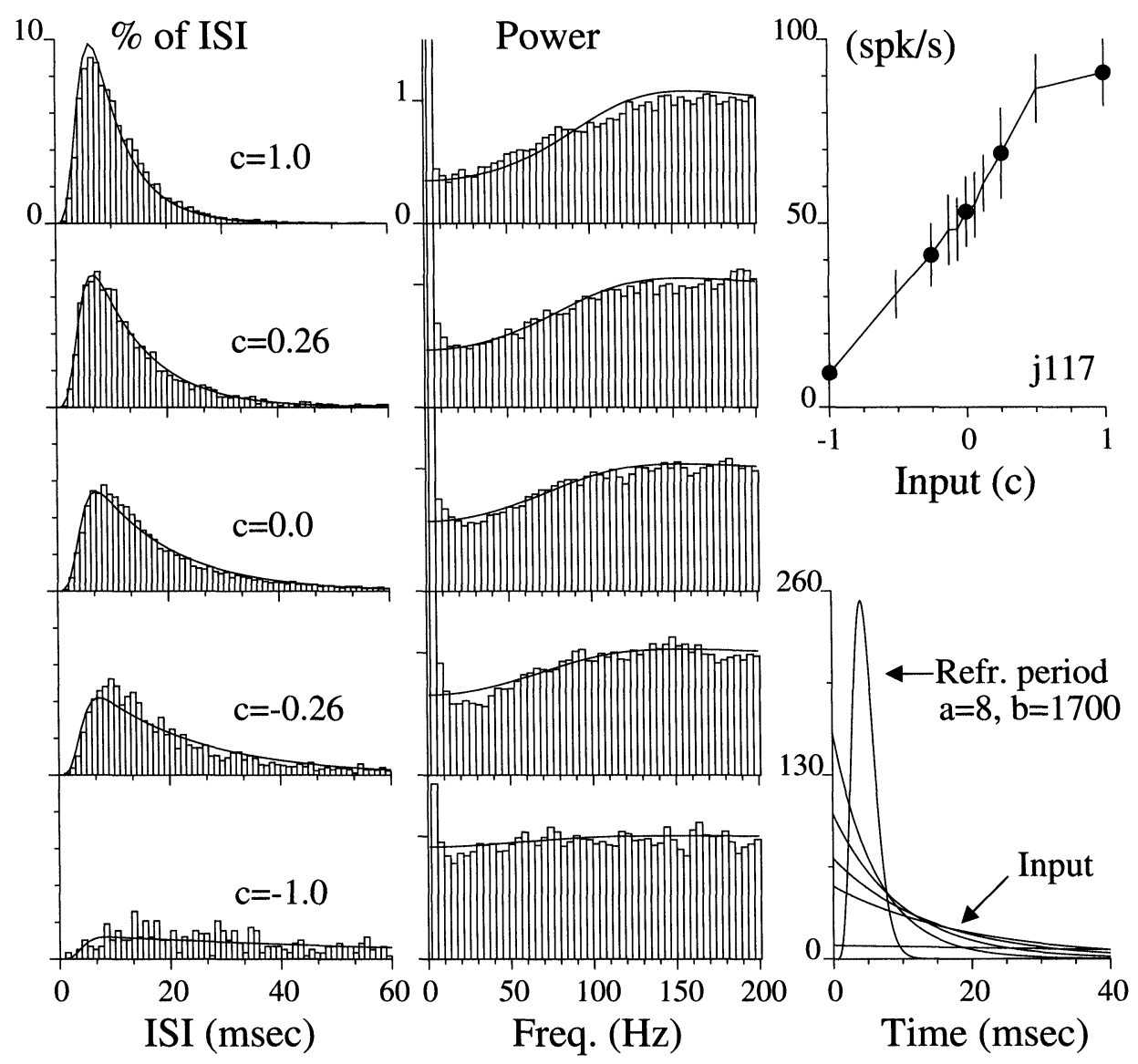

FIG. 2. Neuronal data vs. theory for a neuron ( $j 117)$ with a short refractory period and a broad range of output firing rates. The arrangement is similar to that in Fig. 1 . Under the assumption of a fixed refractory period ( $a=8.0, b=1700.0)$, the left sides of the fits to the ISI histograms (left column) match the data well as firing rate changes (upper right) and even after the firing rate appears to saturate at $c=1.0$. The analytical curves for $\tilde{S}_{x}(\omega)$ (solid lines, center column) give good approximations to the shape of the neuronal power spectrum. (Forty trials of data were used at each stimulus level except $c=0$, where 80 trials were used.)

Some cells tend to fire bursts of action potentials [1], as shown in the top spike train of Fig. 3, which may violate the assumption that the spike train $x(t)$ is a renewal process. The theory can still be applied by considering bursts to be "events" and operating on inter event intervals (IEIs) rather than ISIs. Following Bair et al. [1], an event is defined as the longest train of consecutive action potentials that have no ISIs greater than $d$ msec $(d=8 \mathrm{msec}$ here). We define the process $y(t)$, composed of $\delta$ functions at the center of each event, to be the event train corresponding to the spike train $x(t)$. The event train is plotted beneath the spike train at the top of Fig. 3. The spike train power spectrum (Fig. 3, center) has a substantial peak near $40 \mathrm{~Hz}$, which is not present in the spectrum for events. The IEI histogram (lower left, bars) was fitted to the same mathematical model used in the previous figures, and the analytical power spectra $\tilde{S}_{y}(\omega)$ (smooth curves) from this model match closely those estimated from the event trains. To derive an analytical spectrum for the spike trains, we define $h(t)$ to be the boxcar function (see inset below the event train in Fig. 3) which 

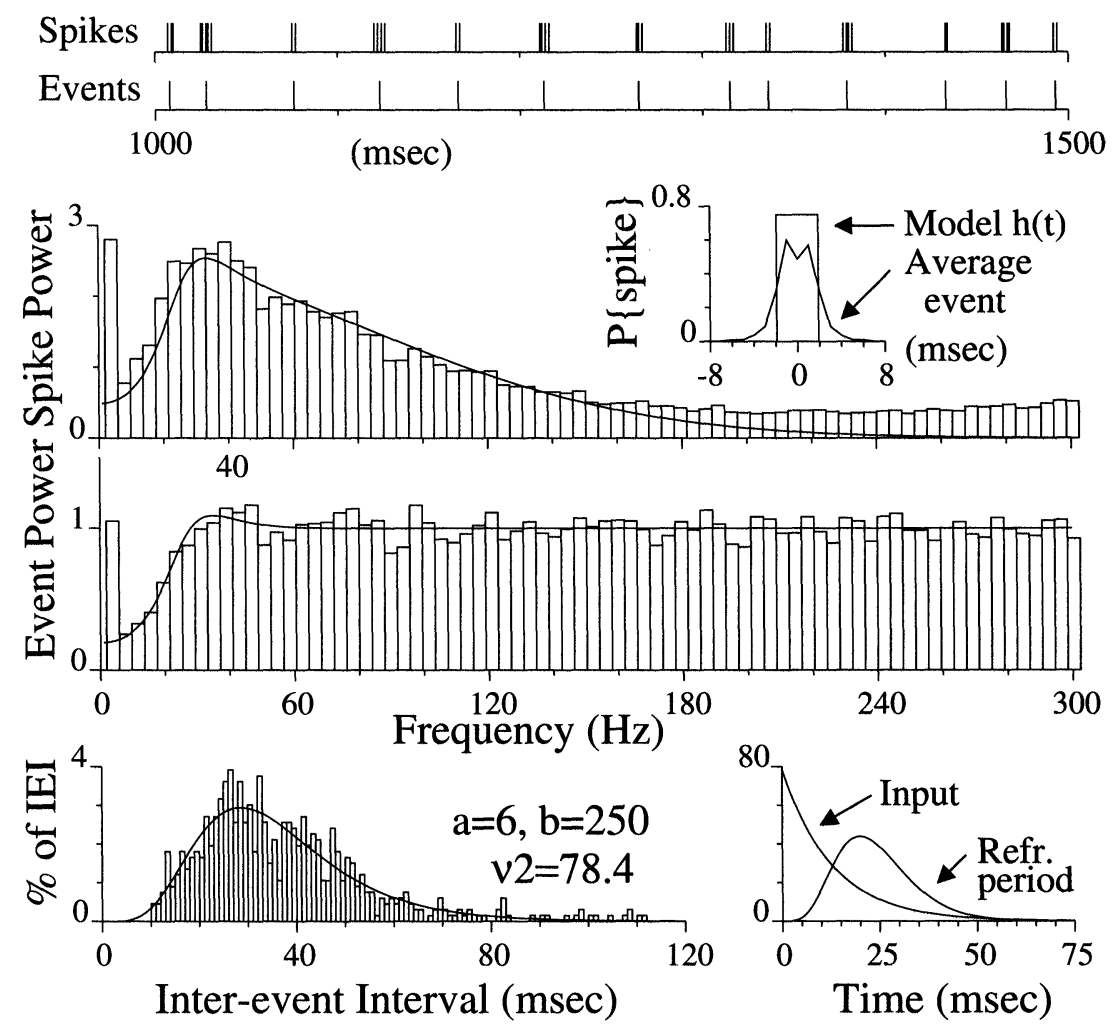

FIG. 3. Modeling bursts of action potentials as single "events" allows the theory to predict the peak in the power spectrum which occurs for cells that fire bursts. The top trace shows a spike train fired by neuron $j 001$. Directly below it is the event train, a series of $\delta$-functions at the center of each event, where an event is defined as the longest train of consecutive action potentials with no ISIs greater than $d$ msec $(d=8$ here). The power spectrum of the spike trains at $c=0.51$ has a peak near $40 \mathrm{~Hz}$ (upper spectrum) while the power spectrum of the corresponding event trains is flat at frequencies higher than $40 \mathrm{~Hz}$ (lower spectrum). This indicates that the large peak observed in the power spectrum is due to the shape, not the timing, of the events. A model of the IEI histogram (lower left) based on a refractory lag and an input lag (lower right) leads to an analytical prediction $\tilde{S}_{y}(\omega)$ (smooth curve) of the event power spectrum. When events are modeled by setting $h(t)$ to the boxcar function shown in the inset below the event train, the analytical expression $\tilde{S}_{x}(\omega)$ for the spike power spectrum predicts the peak observed in the data (upper spectrum). Note that (100) in the Appendix must be modified to account for the boxcar $h(t)$.

serves as a model for the prototypic event. The analytical spectrum, $|H(\omega)|^{2} \tilde{S}_{y}(\omega)$, using a deterministic approximation to the variable event shape, provides an excellent prediction to the location and shape of the peak in the power spectrum. This example shows that the presence of bursts and an interburst refractory period can combine to produce a maximum in the power spectrum.

Fig. 4 demonstrates a case in which a peak in the power spectrum develops from regular firing rather than from burst firing. Again we reduce $x(t)$ to the event trains $y(t)$, fit the model to the IEI histograms (left column), and plot the derived power spectra $\tilde{S}_{y}(\omega)$ against the neuronal spectra (right column). The peak in the event power spectrum is observed to grow as the event firing rate $\nu_{2}$ increases. The values of $\nu_{2}$ for $c=0.0,0.51,1.0$ are approximately 25,50, and 100 events/sec, respectively (see $y$-intercepts of the exponential densities in the inset, Fig. 4). In the limit as 

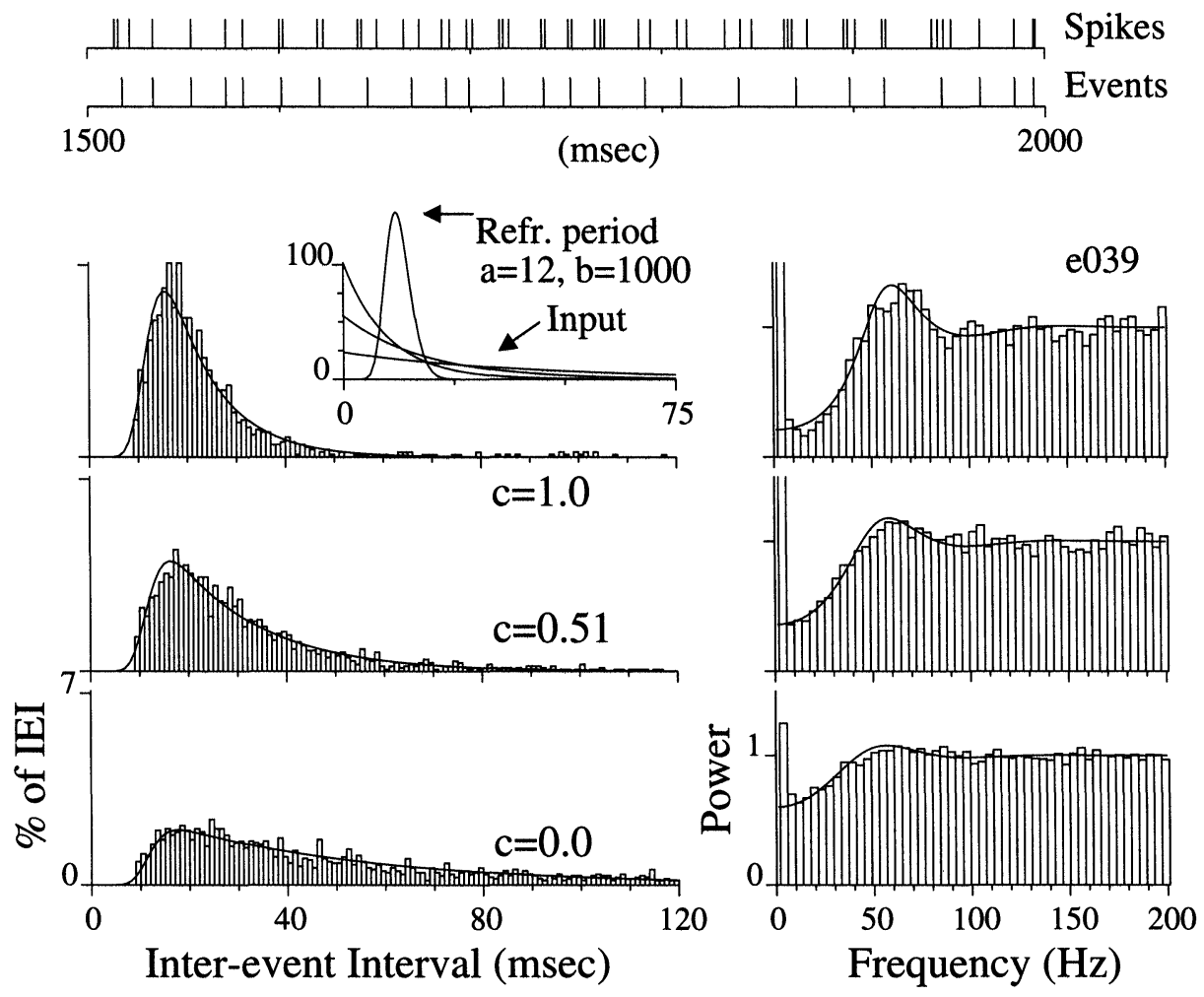

FIG. 4. Spectral peaks due to regular firing are different from those caused by bursts and may occur for a renewal process as the input rate becomes fast with respect to the refractory period. The methods of Figs. 1 and 3 are combined to model the IEI and event power spectrum for neuron e039. The power spectra (bars, right column) are computed for event trains, and the analytical predictions $\tilde{S}_{y}(\omega)$ are based on the $\delta$-function model of events to eliminate the peak due to bursts. As the event rate increases for stronger stimuli, a peak, predicted by the model, develops in the power spectra near the inverse of the mean IEI. Peaks in the power spectra such as these result from the regularity of the timing of the events, rather than from the event shape as seen in Fig. 3.

$\nu_{2} \rightarrow \infty, f(\tau) \rightarrow f_{1}(\tau)$, i.e., the interval p.d.f. becomes the refractory period p.d.f. If $f_{2}(\tau)$ is regular enough to produce a peak in the power spectrum in this limiting case, then we may observe peaks as seen in the right column of Fig. 4. However, if $f_{2}(\tau)$ is, for example, an exponential distribution $(a=1)$, the power spectrum will remain flat even as $\nu_{2} \rightarrow \infty$.

A comparison of the refractory period p.d.f.'s for the four neurons analyzed in the previous figures is shown in Fig. 5 . The means, $a / b$, of the gamma densities range from 4.7-24 msec. Although the means for cell e039 and e085 are 12 and $16 \mathrm{msec}$, their standard deviations are 3.5 and $8.0 \mathrm{msec}$, respectively, indicating the usefulness of having two parameters for the refractory p.d.f. The range of values here are typical of those for the 58 cells analyzed.

3.3. Discussion. Figs. 1 and 2 demonstrate that a fixed stochastic refractory period can be adequate to account for the distribution of interspike intervals across a wide range of firing rates when the input is assumed to induce the neuron to fire at Poisson time instants. (However, Turcott et al. [15] report that the refractory period can change over time in auditory neurons.) The power spectra of the neuronal data in Figs. 1 and 2 are consistent with the analytical curves from the formula derived here, 


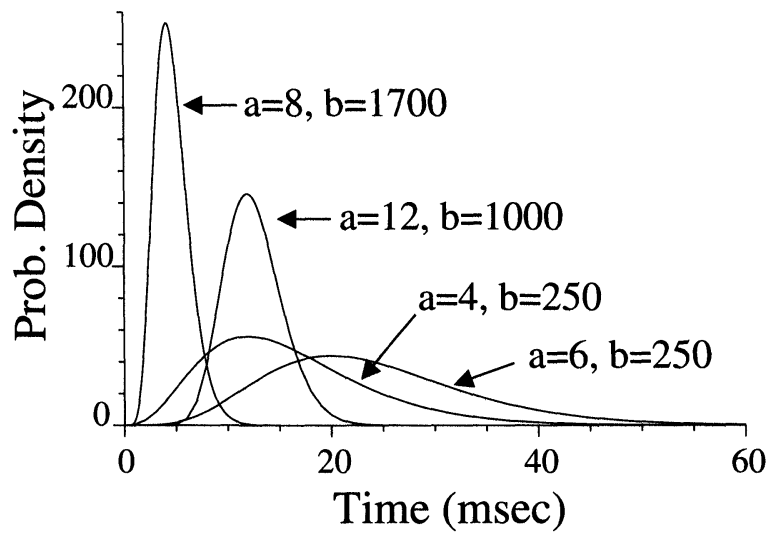

FIG. 5. A comparison of the refractory period densities, $f_{1}(\tau)$, for the four cells studied in the previous figures. Although the mean $\mu_{1}$ and the standard deviation $\sigma_{1}$ of the refractory period often rise or fall together, the middle two examples here have somewhat similar means, 12 and 16 msec, but quite different standard deviations, 3.5 and 8 msec. Recall $\mu_{1}=a / b, \sigma_{1}^{2}=a / b^{2}$.

(8), for the power spectrum of a renewal process with a specified interval density, $f(\tau)$. In particular, the presence of a refractory period causes a dip in the power spectrum at low frequencies (see also [1]).

Figures 3 and 4 show two ways for peaks to arise in the power spectrum of spike trains. First, a peak may arise due to the compound effect of a dip at low frequencies created by a refractory period and the attenuation at high frequencies induced by firing bursts of action potentials (Fig. 3). The frequency of the peak is a function of the refractory period parameters and the shape of the bursts, i.e., events, and is not related to regularity in the inter-event interval. The second type of peak is caused by regularity in the ISI or IEI density, and this regularity is well modeled as the result of an increasing firing rate with a fixed refractory period density. Figure 4 demonstrates this for interevent intervals of a neuron that fires bursts; however, we have observed the same effect in neurons that fire isolated action potentials.

We have presented two mathematical models of spike trains which can lead to peaks near $40 \mathrm{~Hz}$ in the power spectrum based on the formulas derived here and which both incorporate the notion of a refractory period. We emphasize that the concept of refractory period used here is not restricted to a neuron's intrinsic limitations for quickly firing a second action potential; we do not know the presynaptic input to these neurons and cannot distinguish intrinsic from network or input effects which may cause a paucity of short ISIs.

4. Derivations. We will now derive the asserted formula for the neuron firing spectrum, $S_{x}(\omega)$.

LEMMA 4.1. For $\tau \geq 0$ let $f(\tau)$ be bounded and continuous except for a nowheredense set of simple jump discontinuities. Let $f(\tau) \geq 0$, and assume

$$
\int_{0}^{\infty} \tau^{k} f(\tau) d \tau=\left\{\begin{array}{lr}
1 & \text { for } k=0, \\
\mu & \text { for } k=1, \\
\mu^{2}+\sigma^{2} & \text { for } k=2,
\end{array}\right.
$$

with $\mu<\infty$ and $\sigma^{2}<\infty$. Define the Laplace transform

$$
\hat{f}(s)=\int_{0}^{\infty} e^{-s \tau} f(\tau) d \tau \quad(\operatorname{Re} s \geq 0) .
$$


Then

$$
\operatorname{Re} \hat{f}(s)<1 \text { if } \operatorname{Re} s \geq 0 \text { but } s \neq 0
$$

and

$$
\hat{f}(s) \rightarrow 0 \quad \text { as } \operatorname{Im} s \rightarrow \pm \infty \quad(\operatorname{Re} s \geq 0)
$$

and

$$
\int_{-\infty}^{\infty}|\hat{f}(i \omega)|^{2} d \omega=2 \pi \int_{0}^{\infty} f^{2}(t) d t<\infty
$$

Finally, as $s \rightarrow 0$ in the closed right half-plane, we have

$$
\frac{\hat{f}(s)}{1-\hat{f}(s)}=\frac{1}{\mu s}+\frac{1}{2}\left(\mu^{-2} \sigma^{2}-1\right)+o(1) .
$$

On the imaginary axis, as $s=i \omega \rightarrow 0$, we have

$$
\operatorname{Re} \frac{\hat{f}(i \omega)}{1-\hat{f}(i \omega)}=\frac{1}{2}\left(\mu^{-2} \sigma^{2}-1\right)+o(1)
$$

where we may replace o(1) by $O\left(\omega^{2}\right)$ if $\int_{0}^{\infty} \tau^{4} f(\tau) d \tau<\infty$.

Proof. To prove (40), we note that if $s=\alpha+i \omega$ with $\alpha \geq 0$,

$$
1-\operatorname{Re} \hat{f}(s)=\int_{0}^{\infty}\left(1-e^{-\alpha \tau} \cos \omega \tau\right) f(\tau) d \tau .
$$

If $f(\tau)$ is positive and continuous on the interval $a \leq \tau \leq b$, then $f(\tau) \geq$ some $\epsilon>0$ for $a \leq \tau \leq b$, and so

$$
1-\operatorname{Re} \hat{f}(s) \geq \epsilon \int_{a}^{b}\left(1-e^{-\alpha \tau} \cos \omega \tau\right) d \tau \quad(\alpha \geq 0,-\infty<\omega<\infty)
$$

which is positive unless $\alpha=0$ and $\omega=0$. This proves (40).

The assertion (41) follows at once from the Riemann lemma, since $f(\tau)$ is absolutely integrable.

To prove (42), we first note that

$$
\int_{0}^{\infty} f^{2}(t) d t \leq M \int_{0}^{\infty} f(t) d t=M
$$

if $M$ is an upper bound for the bounded p.d.f. $f(t)$. If we now define $f(t)=0$ for all $t<0$, then $\hat{f}(i \omega)$ is the Fourier transform of $f(t)$, and so the identity (42) is just the Parseval-Plancherel identity.

To prove formula (43), we first note that for $\operatorname{Re} s \geq 0$ and $\tau \geq 0$,

$$
e^{-s \tau}=1-s \tau+\frac{1}{2} s^{2} \tau^{2}+\tau^{2} o\left(|s|^{2}\right) \text { as } s \rightarrow 0 .
$$

Now the assumption (38) yields

$$
\hat{f}(s)=1-\mu s+\frac{1}{2}\left(\mu^{2}+\sigma^{2}\right) s^{2}+o\left(|s|^{2}\right) \text { as } s \rightarrow 0 .
$$


Hence,

$$
\begin{aligned}
\frac{\hat{f}(s)}{1-\hat{f}(s)}-\frac{1}{\mu s} & =\frac{-1+(1+\mu s) \hat{f}(s)}{\mu s[1-\hat{f}(s)]} \\
& =\frac{-1+1+\frac{1}{2}\left(\sigma^{2}-\mu^{2}\right) s^{2}+o\left(|s|^{2}\right)}{\mu^{2} s^{2}+o\left(|s|^{2}\right)}
\end{aligned}
$$

so that we deduce formula (43), namely,

$$
\frac{\hat{f}(s)}{1-\hat{f}(s)}-\frac{1}{\mu s}=\frac{1}{2}\left(\mu^{-2} \sigma^{2}-1\right)+o(1) \text { as } s \rightarrow 0 .
$$

On the imaginary axis, if $s=i \omega \neq 0$, we have $\operatorname{Re}(1 / \mu s)=0$. Hence, the required formula (44) follows from (52).

If $f(\tau)$ has a finite fourth moment, then its Laplace transform has the form

$$
\hat{f}(s)=1-\mu s+\frac{1}{2}\left(\mu^{2}+\sigma^{2}\right) s^{2}-\frac{1}{6} m_{3} s^{3}+\frac{1}{24} m_{4} s^{4}+o\left(|s|^{4}\right),
$$

where $m_{3}$ and $m_{4}$ are the third and fourth moments. Then the remainder $o(1)$ in formula (52) may be replaced by something of the form $a s+o\left(|s|^{2}\right)$, where $a$ is a real constant because all the coefficients in (53) are real, so that $\operatorname{Re}(a s)=0$ if $s=i \omega$. Then we obtain

$$
\operatorname{Re} \frac{\hat{f}(i \omega)}{1-\hat{f}(i \omega)}=\frac{1}{2}\left(\mu^{-2} \sigma^{2}-1\right)+O\left(\omega^{2}\right) \text { as } \omega \rightarrow \pm 0 .
$$

LemMA 4.2. Let $f(\tau)$ satisfy the hypotheses of Lemma 4.1. For $t \geq 0$ let $u(t)$ be the unique solution of the Volterra integral equation

$$
u(t)=f(t)+\int_{0}^{t} f(\tau) u(t-\tau) d \tau \quad(t \geq 0) .
$$

Let $\hat{u}(s)$ and $\hat{f}(s)$ be the Laplace transforms of $u(t)$ and $f(t)$. Then

$$
\hat{u}(s)=\frac{\hat{f}(s)}{1-\hat{f}(s)} \quad(\operatorname{Re} s \geq 0, s \neq 0)
$$

and

$$
u(t)=\sum_{k=1}^{\infty} f^{k *}(t) \quad(t \geq 0)
$$

where $f^{k *}(t)$ is the $k$-fold convolution $f(t) * \cdots * f(t)$.

Proof. If we set $u_{1}(t)=f(t)$ and define

$$
u_{n+1}(t)=f(t)+f(t) * u_{n}(t) \quad(t \geq 0)
$$

for $n \geq 1$, we obtain the convergent series solution (57); this is a standard result for Volterra integral equations. A simple majorization argument shows that the solution satisfies

$$
0 \leq u(t) \leq M e^{M t}
$$

since we have assumed $0 \leq f(t) \leq M$ for all $t \geq 0$. 
If we apply the Laplace transformation to both sides of the integral equation, we find $\hat{u}(s)=\hat{f}(s)+\hat{f}(s) \hat{u}(s)$, or

$$
[1-\hat{f}(s)] \quad \hat{u}(s)=\hat{f}(s) \quad(\operatorname{Re} s>M) .
$$

But $\hat{f}(s)$ is analytic for $\operatorname{Re} s>0$ and continuous for $\operatorname{Re} s \geq 0$, and formula (40) of Lemma 4.1 implies

$$
|1-\hat{f}(s)|>0 \quad(\operatorname{Re} s \geq 0, s \neq 0) .
$$

Hence, we may divide (60) by $1-\hat{f}(s)$ and use analytic continuation for Re $s>0$ to deduce

$$
\hat{u}(s)=\frac{\hat{f}(s)}{1-\hat{f}(s)} \quad(\operatorname{Re} s \geq 0, s \neq 0) .
$$

This quotient is analytic in the open right half-plane, and it is continuous in the closed half-plane except at $s=0$, where, by (52),

$$
\hat{u}(s)=\frac{1}{\mu s}+\frac{1}{2}\left(\mu^{2} \sigma^{2}-1\right)+o(1) \text { as } s \rightarrow 0 .
$$

Let us consider the repeated random firing of a single neuron. We call each firing an event $\mathcal{E}$.

In probability theory, the equation (55) is called the renewal equation, and its solution $u(t)$ is called the renewal density. Suppose that an event $\mathcal{E}$, like neuron firing, occurs at successive instants $t_{j}(-\infty<j<\infty)$. For a stationary renewal process we assume that the successive lags $t_{j}-t_{j-1}$ are i.i.d. random variables. If the p.d.f. of each lag is $f(\tau)$, then the associated renewal density $u(t)$ has the following meaning: if an event $\mathcal{E}$ is observed at the instant $s$, then $u(t) d t$ is the probability that some later event $\mathcal{E}$ will occur in an infinitesimal time $d t$ about the instant $s+t$. Thus, $t$ is just the time between two occurrences of $\mathcal{E}$.

The later event may be the first, the second, or in general, the $k$ th event $\mathcal{E}$ after the initial event. Indeed, $f^{k *}(t) d t$ is the probability that the later event is the $k$ th subsequent event, and so the renewal probability $u(t) d t$ is equal to the sum of all the probabilities $f^{k *}(t) d t$ for $k=1,2 \ldots$. That is the meaning of the identity (57).

Example 2. If the independent lags $t_{j}-t_{j-1}=\tau_{j}$ have the common p.d.f. $f(\tau)=$ $\nu e^{-\nu \tau}$, then

$$
\hat{f}(s)=\frac{\nu}{\nu+s}, \quad \hat{u}(s)=\frac{\hat{f}(s)}{1-\hat{f}(s)}=\frac{\nu}{s} \quad(\operatorname{Re} s \geq 0, s \neq 0)
$$

and so the renewal density $u(t)$ is identically equal to the Poisson frequency $\nu$.

Under general conditions, we will show that the renewal density $u(t)$ tends to the average event frequency $\nu$ as $t \rightarrow \infty$. Here we define the frequency $\nu$ as the limit

$$
\nu=\lim _{T \rightarrow \infty} \frac{N}{T}
$$

where $N$ is the number of events $\mathcal{E}$ that occur in time $T$. With probability 1 this limit exists, and

$$
\nu=\lim _{N \rightarrow \infty} \frac{N}{t_{j+N}-t_{j}}=\lim _{N \rightarrow \infty} \frac{N}{\tau_{j+1}+\cdots+\tau_{j+N}}=\frac{1}{\mu},
$$

where $\mu$ is the expected value of every lag $\tau_{k}$. 
We now define $u(t)=u(-t)$ for $t<0$. We will motivate this definition in Lemma 4.4.

LEMMA 4.3. Under the conditions of Lemmas 4.1 and $4.2, u(t) \rightarrow \nu=\mu^{-1}$ as $t \rightarrow+\infty$; and the even function $u(t)$ has the Fourier transform

$$
U(\omega)=2 \pi \nu \delta(\omega)+G(\omega) \quad(-\infty<\omega<\infty),
$$

where $G(\omega)$ is the even, continuous, square-integrable function

$$
G(\omega)=2 \operatorname{Re} \frac{\hat{f}(i \omega)}{1-\hat{f}(i \omega)} \quad(\omega \neq 0), \quad G(0)=\nu^{2} \sigma^{2}-1
$$

Proof. Define the even function $g(t)=u(t)-\nu \quad(-\infty<t<\infty)$. This function has the Laplace transform

$$
\hat{g}(s)=\hat{u}(s)-\frac{\nu}{s}=\frac{\hat{f}(s)}{1-\hat{f}(s)}-\frac{\nu}{s} \quad(\operatorname{Re} s \geq 0),
$$

where we use (44) to define

$$
\hat{g}(0)=\lim _{s \rightarrow 0} \hat{g}(s)=\frac{1}{2}\left(\nu^{2} \sigma^{2}-1\right) .
$$

With this definition, the function $\hat{g}(s)$ is analytic for $\operatorname{Re} s>0$, continuous for $\operatorname{Re} s \geq$ 0 , and square-integrable on the imaginary axis.

For $s=i \omega$ we have the Laplace transform

$$
\hat{g}(i \omega)=\hat{u}(i \omega)-\frac{\nu}{i \omega}=\int_{0}^{\infty} g(t) e^{-i \omega t} d t \quad(-\infty<\omega<\infty) .
$$

But the even function $g(t)$ has the Fourier transform

$$
G(\omega)=\int_{-\infty}^{\infty} g(t) e^{-i \omega t} d t=\int_{0}^{\infty} g(t)\left[e^{i \omega t}+e^{-i \omega t}\right] d t .
$$

Therefore, since $g(t)=u(t)-\nu$ is real valued,

$$
G(\omega)=2 \operatorname{Re} \int_{0}^{\infty} g(t) e^{-i \omega t} d t=2 \operatorname{Re} \hat{g}(i \omega) \quad(-\infty<\omega<\infty)
$$

But formulas (69) and (70) imply, for $s=i \omega$,

$$
\begin{aligned}
2 \operatorname{Re} \hat{g}(i \omega) & =2 \operatorname{Re} \frac{\hat{f}(i \omega)}{1-\hat{f}(i \omega)} \quad(-\infty<\omega<\infty, \omega \neq 0) \\
& =\nu^{2} \sigma^{2}-1 \quad(\omega=0)
\end{aligned}
$$

where (69) implies (74) because $\operatorname{Re}(\nu / s)=0$ for $s=i \omega \neq 0$.

The last three formulas yield the asserted formula (68) for the Fourier transform of the even, continuous, square-integrable function $g(t)=u(t)-\nu(-\infty<t<\infty)$. Formula (67) for the Fourier transform follows because the constant $\nu$ has the Fourier transform $2 \pi \nu \delta(\omega)$. Finally, the Riemann-Lebesgue lemma yields the limit

$$
u(t)-\nu=g(t)=\frac{1}{2 \pi} \int_{-\infty}^{\infty} G(\omega) e^{i \omega t} d \omega \rightarrow 0 \text { as } t \rightarrow \pm \infty .
$$


LEMMA 4.4. For a stationary renewal process, let $u(\tau) \quad(\tau \geq 0)$ be the renewal density discussed in Lemmas 4.2 and 4.3. Let $-\infty<s<t<\infty$, and let ds and $d t$ be differentials about the instants $s$ and $t$. Let $\nu$ be the average frequency of the events $\mathcal{E}$ in the stationary renewal process. Then an event $\mathcal{E}$ occurs in $d s$ with probability $\nu d s$; events $\mathcal{E}$ occur in both $d s$ and $d t$ with probability $\nu u(t-s) d s d t$.

Proof. By stationarity, the probability that an event $\mathcal{E}$ occurs in $d s$ must equal $\nu d s$, where $\nu$ is the average frequency of the occurrences of $\mathcal{E}$. Now the definition of the renewal density implies this for the conditional probability:

$$
\mathrm{P}\{\mathcal{E} \text { occurs in } d t \mid \mathcal{E} \text { occurs in } d s\}=u(t-s) d t \quad(t>s) .
$$

Since $\mathrm{P}\{\mathcal{E}$ occurs in $d s\}=\nu d s$, we deduce

(78) $\mathrm{P}\{\mathcal{E}$ occurs in $d s$ and $\mathcal{E}$ occurs in $d t\}=\nu d s \cdot u(t-s) d t \quad(t>s)$.

In the last formula the function $u(t-s)$ has been defined by the renewal equation only for $t-s>0$. But the joint probability of events in both $d s$ and $d t$ is defined for all $s \neq t$ as a symmetric function. Therefore, for all distinct $s$ and $t$,

$$
\mathrm{P}\{\mathcal{E} \text { occurs in } d s \text { and } \mathcal{E} \text { occurs in } d t\}=\nu d s \cdot u(|t-s|) d t .
$$

If we now define $u(\tau)=u(-\tau)$ for all $\tau<0$, we may write $u(|t-s|)=u(t-s)=u(s-t)$ for all $s \neq t$, and we may use the formula (78) even for $t<s$.

We are now ready to derive the power spectral density of the shot noise generated by the firing instants $\left\{t_{j}\right\}$ and the wave form $h(t)$. For simplicity, assume that $h(t)$ is bounded, continuous, and absolutely integrable. Later it is easy to extend the result to other wave forms such as the Dirac $\delta$-function. (As $h(t)$ approximates the $\delta$-function, its Fourier transform approximates the constant function $H(\omega)=1$.)

THEOREM 4.5. Let $\left\{t_{j}\right\} \quad(-\infty<j<\infty)$ be the random firing instants of the stationary renewal process discussed in the preceding lemmas. Let $h(t)$ be a bounded, piecewise smooth, absolutely integrable function, with Fourier transform $H(\omega)$. Define the associated stationary shot noise

$$
x(t)=\sum_{j=-\infty}^{\infty} h\left(t-t_{j}\right)
$$

Then $x(t)$ has the expected value

$$
\mathrm{E}[x(t)]=\nu \int_{-\infty}^{\infty} h(\tau) d \tau=\nu H(0)
$$

where the instants $\left\{t_{j}\right\}$ have average frequency $\nu$; and $x(t)$ has the power spectral density

$$
S_{x}(\omega)=\nu|H(\omega)|^{2}\left\{2 \pi \nu \delta(\omega)+1+2 \operatorname{Re} \frac{\hat{f}(i \omega)}{1-\hat{f}(i \omega)}\right\}
$$

where $\hat{f}(s)$ is the Laplace transform of the common p.d.f. $f(\tau)$ of the i.i.d. random lags $\tau_{j}=t_{j}-t_{j-1}$. In the last formula, for $\omega$ near zero we assert

$$
1+2 \operatorname{Re} \frac{\hat{f}(\omega)}{1-\hat{f}(\omega)}=\nu^{2} \sigma^{2}+o(1) \quad \text { as } \omega \rightarrow 0
$$


where $\sigma^{2}$ is the lag variance, and where o(1) may be replaced by $O\left(\omega^{2}\right)$ if the lags $\tau$ have a finite fourth moment.

Notation. As usual, we have defined the power spectral density as

$$
S_{x}(\omega)=\int_{-\infty}^{\infty} R_{x}(\tau) e^{-i \omega \tau} d \tau \quad(-\infty<\omega<\infty)
$$

where $R_{x}(\tau)$ is the autocorrelation

$$
R_{x}(\tau)=E\left[x(t) x^{*}(t-\tau)\right] \quad(-\infty<\tau<\infty) .
$$

In our application all the functions $x(t), h(t), \ldots$ are real, but for possible use in other applications, we will do the following derivation for complex-valued functions. We use the asterisk for complex conjugates: $(a+i b)^{*}=a-i b$.

Proof. Let $\phi_{1}(t)$ and $\phi_{2}(t)$ satisfy the same conditions as $h(t)$. Let $\left\{t_{j}\right\}$ be the set of random instants when the recurrent event $\mathcal{E}$ occurs. Then

$$
\mathrm{E} \sum_{j} \phi\left(t_{j}\right)=\nu \int_{-\infty}^{\infty} \phi(t) d t
$$

and, by Lemma 4.4 ,

$$
\begin{aligned}
\mathrm{E}\left\{\sum_{j} \phi_{1}\left(t_{j}\right) \cdot \sum_{k} \phi_{2}\left(t_{k}\right)\right\}= & \nu \int_{-\infty}^{\infty} \int_{-\infty}^{\infty} \phi_{1}(\alpha) u(\beta-\alpha) \phi_{2}(\beta) d \alpha d \beta \\
& +\nu \int_{-\infty}^{\infty} \phi_{1}(\alpha) \phi_{2}(\alpha) d \alpha .
\end{aligned}
$$

The functions $\phi_{1}(t)$ and $\phi_{2}(t)$ are absolutely integrable, and the continuous, even renewal density $u(t)$ tends to $\nu$ as $t \rightarrow \pm \infty$, so that $u(t)$ is bounded. Therefore, the integrals converge.

Now, for $-\infty<\lambda<\infty$, set

$$
\phi_{1}(\lambda)=h(t-\lambda), \quad \phi_{2}(\lambda)=h^{*}(t-\tau-\lambda) .
$$

Then, according to (70), we have

$$
x(t)=\sum_{j} \phi_{1}\left(t_{j}\right), \quad x^{*}(t-\tau)=\sum_{k} \phi_{2}\left(t_{k}\right),
$$

and so the preceding formulas yield

$$
\begin{aligned}
\mathrm{E}[x(t)]= & \nu \int_{-\infty}^{\infty} h(t-\lambda) d \lambda \\
R_{x}(\tau)= & \nu \int_{-\infty}^{\infty} \int_{-\infty}^{\infty} h(t-\alpha) u(\beta-\alpha) h^{*}(t-\tau-\beta) d \alpha d \beta \\
& +\nu \int_{-\infty}^{\infty} h(t-\alpha) h^{*}(t-\tau-\alpha) d \alpha
\end{aligned}
$$

If we replace $t-\lambda$ by $\lambda, t-\alpha$ by $\alpha$, and $t-\beta$ by $\beta$ in the integrands, we get

$$
\begin{aligned}
\mathrm{E}[x(t)]= & \nu \int_{-\infty}^{\infty} h(\alpha) d \alpha \\
R_{x}(\tau)= & \nu \int_{-\infty}^{\infty} \int_{-\infty}^{\infty} h(\alpha) u(\alpha-\beta) h^{*}(\beta-\tau) d \alpha d \beta \\
& +\nu \int_{-\infty}^{\infty} h(\alpha) h^{*}(\alpha-\tau) d \alpha
\end{aligned}
$$


If we take the Fourier transform of $R_{x}(\tau)$, we get

$$
S_{x}(\omega)=\nu H(\omega) U(\omega) H^{*}(\omega)+\nu|H(\omega)|^{2}, \quad(-\infty<\omega<\infty) .
$$

Using the formula (67) for $U(\omega)$, we get

$$
S_{x}(\omega)=\nu|H(\omega)|^{2}\left\{2 \pi \nu \delta(\omega)+1+2 \operatorname{Re} \frac{\hat{f}(i \omega)}{1-\hat{f}(i \omega)}\right\}
$$

where $\hat{f}(s)$ is the Laplace transform of the lag p.d.f. $f(\tau)$. Finally, we use formula (44) from Lemma 4.1 to prove the required formula (83), where $\nu=\mu^{-1}$.

5. Appendix. The spike-rate normalized power spectrum $\tilde{S}_{x}(\omega)$ is derived from formula (8) by setting $h(t)=\delta(t)$, dividing by the mean spike rate, $\nu$, and subtracting the $\delta$-function term. Thus,

$$
\tilde{S}_{x}(\omega)=1+2 \operatorname{Re} \frac{\hat{f}(i \omega)}{1-\hat{f}(i \omega)} \quad(-\infty<\omega<\infty, \omega \neq 0)
$$

where $\tilde{S}_{x}(0)=\nu^{2} \sigma^{2}$, as defined by continuity in equation (10). Let the interval density $f(\tau)$ be defined as the convolution of a gamma density, given in (14), and an exponential density, given in (17), so that its Laplace transform $\hat{f}(s)$ is, as given in formula (20),

$$
\hat{f}(s)=\left(\frac{b}{b+s}\right)^{a} \cdot \frac{\nu_{2}}{\nu_{2}+s} \quad(\operatorname{Re} s \geq 0) .
$$

Then, $\hat{f}(i \omega)^{-1}=\rho e^{i \phi}$, where

$$
\phi=a \tan ^{-1}(\omega / b)+\tan ^{-1}\left(\omega / \nu_{2}\right)
$$

and

$$
\rho=\left(\frac{b^{2}+\omega^{2}}{b^{2}}\right)^{a / 2} \sqrt{\frac{\nu_{2}^{2}+\omega^{2}}{\nu_{2}^{2}}}
$$

Under the previous assumptions and definitions, an explicit expression for $\tilde{S}_{x}(\omega)$ is

$$
\tilde{S}_{x}(\omega)=1+2 \frac{\rho \cos \phi-1}{\rho^{2}-2 \rho \cos \phi+1} \quad(-\infty<\omega<\infty, \omega \neq 0) .
$$

Equation (100) depends on only $a, b$, and $\nu_{2}$ and is the expression for the analytical curves superposed on the neuronal power spectrum histograms in the figures in this paper (except for the top spectrum in Fig. 3, which uses a different definition for $h(t))$.

Acknowledgments. A collaboration between Profs. Christof Koch and William T. Newsome for examining temporal structure in spike trains has motivated this research. We particularly thank Prof. Newsome and Kenneth H. Britten for kindly providing neuronal data for this analysis. W.B. thanks Prof. Koch for technical guidance and inspiration. We are grateful to Martin Stemmler for helpful comments and suggestions. 


\section{REFERENCES}

[1] W. Bair, C. Koch, W. T. Newsome, And K. H. Britten, Power spectrum analysis of bursting cells in area MT in the behaving monkey, J. Neurosci., 14 (1994), pp. 2870-2892.

[2] K. H. Britten, M. N. Shadlen, W. T. Newsome, and J. A. Movshon, The analysis of visual motion: A comparison of neuronal and psychophysical performance, J. Neurosci., 12 (1992), pp. 4745-4765.

[3] D. C. Champeney, Fourier Transforms and Their Physical Applications, Academic Press Inc., New York, 1973.

[4] R. Eckhorn, R. Bauer, W. Jordan, M. Brosch, W. Kruse, M. Munk, and H. J. ReitBOECK, Coherent oscillations: A mechanism of feature linking in the visual cortex?, Biol. Cybern., 60 (1988), pp. 121-130.

[5] G. M. Ghose And R. D. Freeman, Oscillatory discharge in the visual system: Does it have a functional role?, J. Neurophysiol., 68 (1992), pp. 1558-1574.

[6] C. M. Gray AND W. Singer, Stimulus-specific neuronal oscillations in orientation columns of cat visual cortex, Proc. Nat. Acad. Sci. USA, 86 (1989), pp. 1698-1702.

[7] C. M. GRAY, P. KöNig, A. K. ENGEL, AND W. Singer, Oscillatory responses in cat visual cortex exhibit inter-columnar synchronization which reflects global stimulus properties, Nature, 338 (1989), pp. 334-337.

[8] C. HeIDEN, Power spectrum of stochastic pulse sequences with correlation between the pulse parameters, Phys. Rev., 188 (1969), pp. 319-326.

[9] A. K. KREITER AND W. Singer, Oscillatory neuronal responses in the visual cortex of the awake macaque monkey, Eur. J. Neurosci., 4 (1992), pp. 369-375.

[10] T. LukEs, The statistical properties of sequences of stochastic pulses, Proc. Phys. Soc. Lond., 78 (1961), pp. 153-168.

[11] D. H. Perkel, G. L. Gerstein, And G. P. Moore, Neuronal spike trains and stochastic point processes I, the single spike train, Biophysical Journal, 7 (1967), pp. 391-418.

[12] H. P. Press, B. P. Flannery, S. A. Teukolsky, and W. T. Vetterling, Numerical Recipes in $C$, the Art of Scientific Computing, Cambridge University Press, Cambridge, 1988.

[13] M. C. TEICH AND P. DiAment, Relative refractoriness in visual information processing, Biol. Cybern., 38 (1980), pp. 187-191.

[14] M. C. TEICH, L. MATIN, AND B. CANTOR, Refractoriness in the maintained discharge of the cat's retinal ganglion cell, J. Opt. Soc. Amer., 68 (1978), pp. 386-401.

[15] R. G. Turcott, S. B. Lowen, E. Li, D. H. Johnson, C. Tsuchitani, and M. C. Teich, A nonstationary Poisson point process describes the sequence of action potentials over long time scales in lateral-superior-olive auditory neurons, Biol. Cybern., 70 (1994) pp. 209-217. 